\title{
Hacia una enseñanza del locus de enunciación latinoamericano: un acercamiento a partir de los postulados de Juan José Bautista Segales*
}

Fecha de entrega: 7 de noviembre de 2018

Fecha de evaluación: 18 de diciembre de 2018

Fecha de aprobación: 28 de enero de 2019

\author{
Andrés Felipe Suárez López**
}

\section{Resumen}

El presente artículo tiene como propósito presentar una reflexión pedagógica mediante la aplicación de referentes del pensamiento de Juan José Bautista, discípulo de Enrique Dussel. Se busca repensar en términos epistemológicos la enseñanza del locus de enunciación latinoamericano. Se tiene en cuenta que el proceso de desarrollo y consolidación de la razón moderna como forma de compresión de la realidad latinoamericana ha destruido sistemáticamente otras formas de pensar, incluidas las de los pueblos originarios americanos. Es por eso que este artículo busca introducir la reflexión sobre los compromisos que implican pensar y pertenecer a esta zona geográficamente diversa.

Palabras clave: locus de enunciación, capitalismo-modernidad-colonialidad, filosofía de la liberación, horizonte de sentido.

* Esta investigación es producto de los intereses del autor en la filosofía latinoamericana, bajo la intención de ubicar los grandes autores de la disciplina en diálogo con la situación de la problemática regional, especificamente en temáticas pedagógicas. Citar como: Suárez López, A. F. (2019). Hacia una enseñanza del locus de enunciación latinoamericano: un acercamiento a partir de los postulados de Juan José Bautista Segales. Cuadernos de Filosofía Latinoamericana, 40(121), 145-160. DOI: https:// doi.org/10.15332/25005375.5475

* Estudiante de Ciencias Sociales y columnista del medio digital La Oreja Roja. Licenciado en Ciencias Sociales de la Univesidad Antonio Nariño con perfeccionamiento en Licenciatura en Sociología de la Universidad de Sonora, México. Correo electrónico: asuarez107@uan.edu.co 


\section{Toward Teaching the Latin American Locus of Enunciation: An Approach based on the Proposals of Juan José Bautista Segales}

\section{Abstract}

The objective of this article is to present a pedagogical reflection that draws on the thought of Juan José Bautista Segales, a disciple of Enrique Dussel, in order to rethink the teaching of the Latin American locus of enunciation in epistemological terms. It takes into account the fact the process of development and consolidation of modern reason, as the form of understanding Latin American reality, systematically destroyed other forms of thinking, including those of the native American peoples. For this reason, the article seeks to introduce a reflection on the commitments involved in thinking and belonging to this geographically diverse region.

Keywords: locus of enunciation, capitalism-modernity-coloniality, philosophy of liberation, horizon of meaning.

Con los pobres de la tierra / quiero yo mi suerte echar. José Martí

La revolución que será para los pobres no solo será la conquista del pan, sino también la conquista de la belleza, del arte, del pensamiento, y de todas las complacencias del espíritu. José Carlos Mariátegui

\section{Introducción}

El largo siglo $\mathrm{xx}$ ha sido un verdadero desafío para el pensamiento crítico especialmente en las regiones o países donde se ha vivido del modo más duro y crudo el dominio no solo de los imperios de turno, sino del capitalismo tardío y el totalitarismo del modelo neoliberal. La crítica que se hizo desde el marxismo y la izquierda en general 
resultó insuficiente ante el avasallante desarrollo del capitalismo a nivel mundial. Esta situación está mostrando evidencia de que ya no basta con criticar al capitalismo, sino que es necesario al mismo tiempo cuestionar radicalmente el fundamento cultural que lo hizo posible: la modernidad ${ }^{1}$.

Por eso surge la necesidad de comprender los postulados de la filosofía de la liberación desarrollados por Juan José Bautista, como elemento orientador para repensar a nivel epistemológico el proceso de autoconciencia “desde" el locus de enunciación latinoamericano. Estos análisis tienen por su propia naturaleza la capacidad analítica, crítica, reflexiva y las herramientas metodológicas para promover el examen sistemático de la razón moderno-occidental y proponer al mismo tiempo un nuevo horizonte de sentido basado en la vida humana y la naturaleza.

Es por esta razón que entretejer las pedagogías críticas latinoamericanas y el pensamiento de liberación abre nuevos caminos para pensar alrededor de los siguientes cuestionamientos: ¿Qué significa pensar desde el locus de enunciación latinoamericano?, ¿desde dónde se podría hacer una crítica radical al sistema de dominación de la modernidad capitalista-colonial?, y por último, ¿cómo construir un sistema categorial que permita decolonizar a nivel epistemológico las categorías de la razón moderna? Estas preguntas guiarán la discusión a lo largo del artículo.

Por ende, y considerando estas preguntas, el texto se va dividir en cuatro secciones que versan principalmente sobre el trabajo de investigación hasta ahora realizado. En su orden: 1) el Marx de Enrique Dussel y la revolución teórica de Juan José Bautista, 2) hacia un concepto decolonial del locus de enunciación latinoamericano, 3) ¿qué significa pensar desde América Latina?, y 4) hacia una enseñanza del locus de enunciación latinoamericano.

La intención de pensar "desde" este tipo de realidades no occidentales y "desde" la exterioridad del no-ser de la totalidad capitalista tiene como objetivo, en sentido estricto,

1 En este texto el término modernidad será entendido en su relación constitutiva con el capitalismo y la colonialidad. Estará pues referido esquemáticamente a la triada modernidad-capitalismo-colonialidad, que tiene su origen en el "descubrimiento" de América y la posterior industrialización de Europa gracias a la apertura de vías comerciales transatlánticas. Dicha modernidad no hubiera sido posible sin el movimiento intelectual europeo del siglo XVI al XIX, el cual construye una justificación supuestamente racional alrededor de la violencia ejercida contra los pueblos originarios amerindios. 
reflexionar sobre aquellos planteamientos teóricos, ético-políticos y metodológicos que fundamentan las posiciones eurocéntricas, colonialistas, capitalistas, patriarcales y racistas que aún erosionan el pensamiento y la enseñanza en América Latina y que impiden construir un horizonte de sentido "más allá de modernidad".

\section{El Marx de Enrique Dussel y la revolución teórica de Juan José Bautista}

La revolución teórica de Juan José Bautista ${ }^{2}$ está relacionada directamente con el proceso intelectual de Enrique Dussel y su relectura de Marx en los años ochenta y noventa. Durante este periodo Dussel decide estudiar al pensador alemán cronológicamente desde los textos inéditos en alemán. Allí se da cuenta de que El capital, obra magna de Marx, tuvo antes de su publicación en 1867 cuatro redacciones previas, cada una de ellas distinta, con avances de orden teórico y epistemológico que merecían ser estudiados. En efecto, allí estaba reseñado, por un lado, el proceso critico que llevo a Marx a escribir El capital (el llamado método dialéctico), y por el otro, la identificación de categorías y nociones desconocidas por el marxismo del siglo xx.

A raíz de esto el problema del método tendría una importancia central pues el marxismo del siglo xx (en el que se formó Juan José Bautista) desconocía el potencial teórico de los borradores anteriores a El capital -identificados por el $\mathrm{Mega}^{3}$ como los Grundrisse, los manuscritos del 61 al 63 y los manuscritos del 63 al 66-, todos ellos con referencias metodológicas fundamentales para el avance teórico del llamado giro decolonial. Sin embargo, Dussel intuía que se necesitaba estudiar nuevamente a Marx

2 Pensador boliviano. Hizo estudios con Hugo Zemelman en el Colegio de México entre 1988 y 1991. Luego estudió Filosofía desde la licenciatura y posteriormente hizo la maestría en la Facultad de Filosofia de la Unam. Se convertiria en discipulo de Enrique Dussel y Franz Hinkelammert. En 2015 fue galardonado con el Premio Libertador al Pensamiento Crítico por su obra "¿Qué significa pensar desde América Latina?".

3 Marx-Engels-Gesamtausgabe (Mega), proyecto de publicación que ha subdividido la obra entera de Marx y Engels en cuatro secciones. La primera (32 tomos) incluye trabajos, artículos y borradores de ambos pensadores, anteriores y paralelos a las redacciones de El capital. La segunda (15 tomos) está dedicada íntegramente a lo que Marx escribió para El capital, desde los Grundrisse de 1857-58 hasta los documentos de 1881-82, además de las ediciones que Engels preparó para la publicación de los tomos II y III de dicha obra, hasta 1894. La tercera (35 tomos) está dedicada a la correspondencia integra de ambos. La última sección (32 tomos) contiene artículos y notas de ambos autores. En total suman 114 tomos. 
debido a las tergiversaciones mecanicistas y malas interpretaciones, todas ellas sintetizadas en la producción teórica del francés Louis Althusser y los manuales soviéticos de K. Kautsky, Mijaíl T. Iovchuk y Teodor I. Oizerman. Bautista afirma:

Quienes se auxilian de los manuales, casi siempre terminan mal parados. Apropiarse de la obra del genio de Marx implica comprender antes, o paralelamente muchísimos presupuestos teóricos, políticos, económicos, teológicos, históricos, filosóficos, literarios, etc., que Marx los da por supuesto en la redacción definitiva de El capital. (2018, p. 1)

A este contexto se le adhiere la avasallante aparición de nuevos problemas, fenómenos y sujetos políticos en América Latina, que para Dussel eran los llamados a configurar una nueva subjetividad en la región y cuya emergencia abriría nuevos campos teóricos en las ciencias sociales. Para ese momento Juan José Bautista, marxista secular y licenciado en filosofía de la Universidad Mayor de San Andrés en Bolivia, motivado por las guerras del agua y del gas que acontecieron en su país entre 2001 y 2003, decide viajar a México para participar del seminario de Dussel en la Unam, donde más tarde recibiría su doctorado. Dussel proporcionaría una aproximación sistemática a la obra de Marx, y lo haría desde el locus de enunciación latinoamericano, lo que impulsó a Bautista a seguir la ruta trazada por estos avances teóricos.

En 1975, al llegar a México, Dussel se propone pensar sobre problemas relativos al subdesarrollo, la marginación y la pobreza en América Latina. Por eso Marx y su sistema categorial valían como punto de análisis. No obstante, su interpretación de Marx prefería la noción de pueblo y no la de clase social ${ }^{4}$, cuestión que molestó a los marxistas dogmáticos, que hasta ese momento solo habían leído la última redacción del El capital y la literatura soviética:

Dussel se puso a estudiar a Marx “Marx”, o sea a Marx mismo, para apropiarse
de su lenguaje y categorías, pero no a los marxistas, comentadores de moda,
o lectores de Marx que en ese entonces abundaban hasta por las facultades de
ciencias exactas. (Bautista, 2018, p. 6)

4 En este sentido el marxismo del siglo xx siendo clasista, dejó de ser popular, por eso se quedó desde fines del ochenta sin sujeto de la revolución. Autores como Ernesto Laclau, Edgar Morin, Michael Foucault, Toni Negri, etc., apartándose de Marx y su método, no lograron hacer una crítica de la totalidad, y prefirieron generalizar otras especificidades. 
Como resultado, entre 1985 y $1993^{5}$ Enrique Dussel publicó cuatro libros de investigación exhaustiva sobre la obra de Marx. Ninguno recibiría la debida atención hasta 2001, cuando se tradujeron al inglés Hacia un Marx desconocido. Un comentario de los manuscritos del 61-63 y al italiano El último Marx (1863-1882) y La liberación latinoamericana. En este mismo sentido, libros como La producción teórica de Marx. Un estudio de los Grundrisse de 1985 y los tres ya mencionados pasarían desapercibidos para las viejas generaciones de marxistas latinoamericanos y el marxismo occidental. Luego de presentar una visión de conjunto de los manuscritos de El capital, Dussel publica su cuarto libro sobre el filósofo de Tréveris: Marx y la modernidad (1995), donde se presenta por primera vez la relación de los manuscritos ya no solo con el capitalismo, sino con la crítica de la modernidad colonial.

Después de haber comprendido el método crítico-dialéctico utilizado por Marx para escribir El capital Dussel aseguró que aquel todavía tendría mucho que decir en la actualidad porque habría distinguido dos tipos específicos de capital, el central y el periférico, que no son el capital en general (Bautista, 2018, p. 17). Esta hipótesis presupuso un avance que el marxismo del siglo xx no entendió, pero que Dussel tematizó como la competencia de capitales entre sí o la cuestión de la dependencia. Es decir, la competencia adquiriría una dimensión dialéctica, ya no solo reflejada entre la contradicción capital-trabajo, sino también entre dos capitales en abstracto: el del primer mundo y el del tercero. En este caso la contradicción aparece cuando los capitales primermundistas explotan inclusive a los capitales tercermundistas, con menos composición orgánica y menor desarrollo tecnológico. Explotación de la cual se benefician hasta los obreros del primer mundo, que explotan así indirectamente a sus pares del tercero. Marx afirmaría:

Si el gran capitalista quiere desplazar al pequeño, tiene frente a él todas las ventajas que el capitalista en cuanto capitalista tiene frente al obrero. La mayor

5 Es preciso aclarar que entre los ochenta y los noventa, mientras Dussel hizo la recepción de toda la obra de Marx en torno a El capital recogida en la segunda sección del Mega, no estaban aún publicados los tres volúmenes de los Hauptmanuskripts -esto es, la tercera redacción de El capital-, correspondientes al tomo 4 y titulados "Manuscritos económicos de 1863-1867". Tampoco estaban publicados los tomos 10,11, 12, 13 y 15, que correspondian a la cuarta edición del tomo I de 1890, los manuscritos para el tomo ॥ hasta 1881, los manuscritos para el tomo III escritos entre 1871 y 1882 y la versión de Engels del tomo III de 1894. Por ello Dussel insiste en que varias veces visitó el archivo holandés donde se hallaban muchos de estos manuscritos aún inéditos para poder trabajar Marx directamente en sus originales. 
cantidad de su capital le compensa de los menores beneficios e incluso puede soportar pérdidas momentáneas hasta que el pequeño capitalista se arruina, y él se ve libre de esta competencia. Así acumula los beneficios del pequeño capitalista. (1932, p. 80)

En este mismo sentido, el Marx de Dussel tiene un carácter novedoso cuando este desarrolla la diferencia entre el método de Hegel y la inversión del método de Marx. Una discusión que en autores de la talla de G. Lukács, K. Korsh, H. Marcuse, K. Kosík, L. Althusser y J. Habermas (connotados nombres del marxismo occidental) no era lo bastante clara. Esto debido al desconocimiento de la obra completa de Marx y las cuatro redacciones de El capital, en las que se hacen descripciones metodológicas de suma importancia para el avance del proyecto científico marxista.

Tal diferencia aparece porque Marx parte del no-ser o la exterioridad de la totalidad. Esto significa partir del análisis de lo que el Ser o la totalidad excluyen, marginan, condenan, encubren o invisibilizan. Bautista afirma que, por el contrario, "Hegel parte de la totalidad, o del Ser, esto es, de la realidad moderna que como horizonte ha creado el capitalismo de su tiempo, es decir, de la realidad objetivada por el capitalismo" (2018, p. 23). La diferencia radica en la oposición entre el Ser-capitalista y el no-ser-excluido, la cual se va a encontrar tanto en el pensamiento de Marx y Dussel, como en el de su discípulo, Bautista, quien usa esta distinción como su locus de enunciación para repensar el no-ser latinoamericano.

A la luz de estos avances en lo metodológico, la crítica a la economía política efectuada por Marx sigue siendo actual. Y también lo es la segunda crítica, la del sistema de las categorías de la modernidad capitalista-colonial hecha por Dussel a partir de un componente ético-crítico. Gracias a esta recepción de la obra de Marx, Bautista ha logrado dirigir sus reflexiones a otros problemas relacionados con la trasformación y revolución que están proponiendo los nuevos actores políticos y sujetos sociales excluidos. En efecto, ahora estos impulsan una praxis liberadora de los pueblos indígenas y demás movimientos populares, sociales y comunitarios que efectivamente ejercen el poder delegado y obediencial desde el locus de enunciación latinoamericano. Bautista afirma:

Por ello es que desde el principio intuimos que el Marx de Dussel, no era el mismo Marx del marxismo occidental, ni mucho menos del marxismo estándar, porque no partía de los mismos presupuestos de estos, es decir, no asumía 
ingenuamente a la modernidad como fundamento, pero tampoco presuponía los problemas de los países de primer mundo ingenuamente, ni parte sin tematización previa de supuestos como la concepción [sic] de realidad, ciencia e historia que manejó el marxismo del siglo xx casi naturalmente, los cuales tomó de Europa y la modernidad como si fuesen evidentes en sí mismo. (2018, p. 2)

Esto significa, en términos epistemológicos, un logro teórico comparable al de una revolución. Bautista, discípulo de Dussel, comprendió que todo conocimiento se piensa de cara a la realidad, siempre dialogando con los grandes pensadores, siempre teniéndolos como interlocutores a partir de los cuales se construyen y afinan categorías para expresar del mejor modo posible, analíticamente hablando, la realidad que se está pensando. Que en este caso es la miseria del pueblo latinoamericano, la producción a escala mundial de la explotación y la colonización creciente de los que se sitúan en el no-ser de la totalidad capitalista. Es por esto que criticar a la modernidad desde América Latina ha sido para este filósofo andino una de sus preocupaciones principales pues es desde aquí, en el sur global, donde se afinca la posibilidad de pensar "más allá de la modernidad".

\section{Hacia un concepto decolonial del locus de enunciación latinoamericano}

Hecha la descripción del Marx de Dussel y la revolución teórica que supuso esta lectura en Bautista, es preciso preguntarse en este apartado por aquello que el filósofo boliviano ha denominado locus de enunciación latinoamericano, para comprender sucintamente el aporte epistemológico de esta categoría. Por lo pronto, el giro decolonial la usó para criticar a fondo el sistema categorial de la modernidad capitalista-colonial, la cual, como se sabe, ha construido ciencia, historia, filosofía, ética, política y literatura basada en el eurocentrismo y la exclusión de cualquier "otra" forma de pensamiento que provenga de límites geográficos marginales:

La ciencia y la filosofía modernas no son más que racionalizaciones de sus propios prejuicios, los cuales, gracias a la forma argumentativa que han producido ellas, aparecen como sumamente racionales, esto es, inteligibles y con sentido, perfectamente válidas para Occidente, pero con escasa validez o sentido cuando 
se las piensa desde otros horizontes culturales y civilizatorios. (Hinkelammert, 1991, p. 23)

Así mismo, para Bautista el pensamiento moderno-capitalista del primer mundo construyó un relato histórico al que se le dio carácter universal, lo que produjo la negación y el olvido del pensamiento andinoamazónico, tildado de premoderno y bárbaro. En el nombre de la racionalidad, la ciencia y la filosofía la modernidad afirmó su propio privilegio sobre otras formas de racionalidad o sobre formas de pensamiento que desde su perspectiva fueran irracionales. Esto indujo injustificadamente a los intelectuales latinoamericanos a dar la espalda a su realidad, en la que la miseria, la pobreza, y el despojo eran el común denominador (Mignolo, 2005). Sin darse cuenta estaban pensando desde el marco categorial que los explotaba a ellos y a su propio pueblo:

La modernidad aparece cuando Europa se afirma como el "centro" de una historia mundial que inaugura; la "periferia" que rodea este centro es entonces parte de su autodefinición. La oclusión de esta periferia (y del papel de España y Portugal en la formación del sistema mundial moderno desde finales del siglo Xv hasta mediados del XVII) lleva a los principales pensadores contemporáneos del "centro" a una falacia eurocéntrica en su comprensión de la modernidad. Si su comprensión de la genealogía de la modernidad es parcial y provincial, sus intentos de crítica o defensa son igualmente unilaterales y, en parte, falsos. (Dussel, 1993, p. 45)

Es por esto que descentrar el punto cero ${ }^{6}$ del pensamiento dominante es fundamental para pensar de cara a la realidad: gracias al movimiento dialéctico de esta, que es siempre cambiante, muchos actores sociales de origen campesino e indígena en América Latina están empezando a emerger en el escenario político. Cabe señalar que algunos de ellos todavía muestran un amplio margen de exterioridad cultural y civilizatorio respecto a la totalidad de la modernidad-capitalista.

6 Este concepto se extrae del libro de Santiago Castro-Gómez La hybris del punto cero. En este se parte del supuesto de que la violencia ejercida por el colonialismo europeo en el mundo no fue solo física y económica sino también epistémica. La tesis se desarrolla considerando la historia desde el siglo XvI, momento en el cual muchas formas de conocer quedan integradas en una jerarquía cronológicocognitiva donde el conocimiento científico-ilustrado aparece en el lugar más alto mientras todas las demás epistemes son vistas como formas de barbarie. El punto cero de todo conocimiento estaría posicionado en Europa, y su dispositivo de legitimación y poder seria la violencia. 
Esto supone para la ciencia social contemporánea y para su enseñanza un desafío importante pues pensar desde el locus de enunciación latinoamericano ha permitido, en cierto sentido, criticar a la modernidad capitalista-colonial. Además, esa crítica permite construir un marco categorial de la razón poscolonial, a través del cual grupos diversos con prácticas teóricas múltiples han logrado manifestar, a raíz de las herencias coloniales y desde su lugar de origen, la historia moderna-capitalista de dominación y las historias contramodernas coloniales de una manera crítica y propositiva.

El locus de enunciación latinoamericano, como categoría poscolonial ${ }^{7}$, no solamente hace inteligible, pensable y posible cuestionar la modernidad capitalista-colonial, el modelo neoliberal o incluso el socialismo real del siglo xx, sino al mismo tiempo problematizar y criticar la racionalidad que los presupone y les da sentido. Para Bautista si de lo que se trata es de pensar la realidad latinoamericana desde esta misma como lugar de origen es indispensable mantener en equilibrio dos pretensiones. La primera es producir una concepción de economía, ética y política poscapitalista, comunitaria o transmoderna, cuyo horizonte teleológico permita elevar al plano de lo concreto las necesidades e intereses de las subjetividades políticas excluidas por la modernidad capitalista-colonial. En segundo sentido, y paralelamente, hay que producir un nuevo sistema categorial con conceptos y significaciones que hagan inteligible el proyecto en mención, con el fin de materializar en lo concreto toda significación "autentica" que aparezca en el locus de enunciación latinoamericano.

Ahora bien, es pertinente no perder de vista que el locus de enunciación es un concepto que se conjura para fundamentar una crítica no europea de la modernidad capitalistacolonial. Ser latinoamericano supone un proceso histórico-cultural determinado por un modelo ideal ${ }^{8}$ diferenciado, es decir, diverso en tanto se afirma como pensamiento del no-ser de la totalidad capitalista. Situarse como diferente, como de la periferia, tiene como consecuencia política criticar

7 El prefijo "pos" tiene aqui una doble intensión: por un lado, relacionada con el concepto de precedencia que determina un "estar" en el tiempo presente, y por el otro, señalar la condición de razón anticolonial surgida después de la independencia política y del desprendimiento de la colonización europea.

8 Para Franz Hinkelammert el concepto de modelo ideal designa los mitos o utopias presupuestas en todo locus de enunciación de cualquier origen geográfico. Esto quiere decir que toda forma de pensamiento, venga de donde venga, siempre tiene una matriz de conocimiento que la hace diferenciada, diversa. 
el trauma, la inconsciente falta de respeto, la ignorancia - por quienes hablan de derechos humanos y de modernidad- de cómo se siente el ninguneo que los valores de progreso, de bienestar, han impuesto a la mayoría de habitantes del planeta, que en este momento tienen que re-aprender a ser. (Mignolo, 2005, p. 20)

En síntesis, posicionarse desde el no-ser de la totalidad capitalista es, según Bautista, un desafío necesario para responder como latinoamericanos a las grandes preguntas de la filosofía - tal como proponía Hugo Zemelman-.

Dicho esto, responder a la pregunta “¿Qué es el locus de enunciación latinoamericano?" parece un tema muy complicado. Es de suponer que hasta este momento intelectuales latinoamericanos como E. Dussel, W. Mignolo, S. Castro-Gómez, R. Grosfoguel, J. J. Bautista, etc., están haciendo una crítica sistemática del marco categorial de la modernidad capitalista-colonial y sus dispositivos de poder ${ }^{9}$. Solo después de eso podrán responder desde este lugar geográfico preguntas como “¿Qué significa pensar desde América Latina?” o “¿Qué implicaciones tiene enseñar el significado político poscolonial del locus de enunciación latinoamericano desde su lugar de origen?”. Esta cuestión se ampliará en los siguientes apartados.

\section{¿Qué significa pensar desde América Latina?}

Llegados hasta este punto es preciso establecer la relación entre el locus de enunciación latinoamericano y el significado de pensar desde ese lugar de origen. La respuesta a esta cuestión se encuentra en el terreno de la filosofía. De ahí que sea necesario interrogar acerca del sentido de esta hoy. Para Bautista tal sentido se encuentra en la condición de posibilidad del pensar mismo. Ahora bien, ¿qué significa pensar que la vida es condición de posibilidad del pensar? O planteado de otro modo, ¿sería posible el pensar sin la vida? Estas preguntas obligan a cambiar la forma de tematización del pensar porque la realidad que las motivó ha cambiado y sus lugares de origen también (Bautista, 2014).

9 Esta expresión es tomada de la lectura que hace Luciana Cadahia (2016) de Michel Foucault y Judith Revel. El dispositivo puede ser concebido como un operador material de poder que produce técnicas, estrategias y formas de sujeción y subjetivación. 
Cuando se piensa desde América Latina, la tematización empieza a ser otra. Esto significa que el pensar ya no parte de su sentido positivo, como acción instrumental para comprender e interpretar el mundo en el que vivimos (abstracción de la razón moderna), sino de su radicalidad: el pensar mismo se preocupa por aquello que lo hizo posible, y esto es, para Bautista, la producción de vida. Dicho de otro modo, para que sea posible el pensar primero debe existir la vida. Por eso esta no puede estar al servicio de aquel, sino al contrario. Solo así siguen siendo posibles la vida humana y la vida de la naturaleza. Bautista afirma:

Por eso es que [...] las razones y las palabras no se pueden separar de quienes las piensan y las dicen. Así las palabras y las razones son humanas porque son mundanas, siempre se despliegan en un horizonte histórico de sentido, que llamamos mundo de la vida. (2005, p. 2)

Nuevamente, ¿qué significa pensar? Esta pregunta, en su formulación clásica, fue problematizada en la década de los cincuenta por Martin Heidegger (2005) en su libro ¿Qué significa pensar?. Para él, el problema que merecía pensarse en aquella época era la incapacidad de la ciencia natural y la lógica matemática de pensar sobre su propia incapacidad reflexiva, en términos de Heidegger, sobre su propia carencia de conciencia racional ${ }^{10}$ :

Heidegger afirmaba esto en una época en la cual la razón calculadora (o sea, matemática, la que ha desarrollado con bastante éxito la ciencia natural y la tecnología como cuantificación de la realidad) se imponía en el mundo y en la academia de tal modo que ya estaba empezando a desplazar y a negar las reflexiones relativas a las consecuencias negativas que cualitativamente produce la civilización dominada por la técnica y la tecnología. (Bautista, 2014, p. 79)

Esta premisa da pie para introducir la respuesta, que para Bautista se desarrolla alrededor de la discusión de la existencia latinoamericana (locus de enunciación), de su sentido para la vida o para la muerte.

10 Heidegger agrega: "Todavía no hemos llegado ante el ámbito, todavia no hemos entrado en el ámbito de lo que de suyo quisiera ser pensado en un sentido esencial. Posiblemente esto tenga su raiz en que nosotros, los hombres, todavía no nos dirigimos suficientemente a lo que quisiera ser pensado [...]. El hecho de que no pensemos de ninguna manera se debe a que el hombre todavía no se dirige en medida suficiente a lo que originalmente quisiera ser pensado" (2005). 
Estrictamente hablando el pensar se desarrolla a partir del cuestionamiento de lo que nos preocupa, lo que da que pensar. Por eso preguntarse qué es aquello que da que pensar es fundamental para precisar lo que es digno de ser pensado. Ahora, lo que da que pensar es aquello que es grave. Esto quiere decir que el pensar habitualmente es motivado por la gravedad de un problema. Por eso para que el pensar acontezca, la razón tiene que abocarse a tematizar, o sea problematizar, lo que es grave. Pero solo de acuerdo a la gravedad de lo tematizado puede la razón profundizar en el acto de pensar (Bautista, 2014). A la luz de esto, tenemos dos preguntas que guían el debate: ¿Qué es lo gravísimo de nuestra época que da que pensar? Y ¿cómo podemos pensar de tal modo que iluminemos con sentido vital posibles salidas a la situación gravísima y crítica que nos ha motivado a pensar?

Debe precisarse que lo gravísimo del presente tiene dos sentidos. El primero es, el origen de la modernidad europeo-occidental, que destruye sistemáticamente las dos únicas fuentes a partir de las cuales es posible producir y reproducir cualquier forma de vida: la naturaleza y el trabajo humano. El segundo, constitutivo del otro, es el marco categorial proveniente de Europa y Estados Unidos, que da sentido y legitima ese horizonte cultural basado en la muerte, la exclusión y la pobreza en el mundo:

\begin{abstract}
Alain Touraine, sociólogo francés, decía [...] que después de una larga evaluación, se había dado cuenta de que la ciencia social del siglo xx había pensado la realidad del siglo xx con categorías del siglo xIX, en cuyos contenidos nunca había estado problematizada o tematizada América Latina como problema. Es decir, que en el contenido problemático presupuesto en la formalización de los conceptos y categorías con los cuales la ciencia social piensa o tematiza los problemas, nunca había estado la realidad latinoamericana, sino solo la realidad europea, norteamericana o del primer mundo del siglo xIx. (Bautista, 2014, p. 83)
\end{abstract}

Por eso es menester pensar esos dos sentidos como condiciones gravísimas del presente, y desde América Latina producir otra idea del significado del pensar, basada en el locus de enunciación latinoamericano, cuya característica fundamental es el noser o el nivel de exterioridad de la totalidad capitalista. El sentido del pensar de esta época es en definitiva pensar desde aquello que se negó, despreció, ocultó; se condenó al olvido y cuyo resultado fue la pobreza, la miseria y la exclusión de cualquier otra forma de pensamiento. 
Por consiguiente, la respuesta a la pregunta “¿Qué significa pensar desde América Latina?" tiene, una sola respuesta, que para Bautista se resumiría de la siguiente manera: es un proceso de autoconocimiento del pensar, que se da desde el no-ser de la totalidad capitalista, a partir del cual la pobreza, la miseria y la exclusión se materializan como condición necesaria para el análisis crítico de la realidad latinoamericana. Realidad que históricamente se encuentra determinada por el horizonte de sentido que impuso la modernidad capitalista-colonial a través de la violencia y la exclusión. Un horizonte de sentido que esta destino a trascender (transmodernidad ${ }^{11}$ ) para preservar aquello que da sentido a todo: la vida.

\title{
Hacia una enseñanza del locus de enunciación latinoamericano
}

\begin{abstract}
Ahora bien, estas reflexiones no tendrían sentido sin la articulación de la dimensión educativa pues, en cierto modo, la descolonización epistemológica está atravesada por el ejercicio popular. Es por esto que el problema que da que pensar en este apartado es el de la relación entre el locus de enunciación latinoamericano y la educación. Sin un proceso de enseñanza liberador, los principios clave de la inteligibilidad de la razón moderna condenarían a las subjetividades del no-ser al vaciamiento de su locus de enunciación, cosa que imposibilitaría ir "más allá de la modernidad”.
\end{abstract}

Por eso se hace necesario descolonizar la perspectiva educativa que actualmente opera en América Latina, la cual se encuentra determinada, en primer lugar, por la relación unidireccional maestro-alumno, y en segunda instancia, por el control de la subjetividad a través de la institución que comúnmente conocemos como escuela. Estas dos determinaciones apuntan a un objetivo común: ocultar que en los límites trazados por la modernidad capitalista-colonial otras formas de enseñar y pensar están aconteciendo paralelamente desde el no-ser en el presente.

Por consiguiente, la autoconciencia con respecto al locus de enunciación latinoamericano es fundamental para conocer las pretensiones pedagógicas que se persiguen. De

11 Enrique Dussel define la transmodernidad como un proyecto que parte de más allá de la modernidad, cruzándola de modo transversal a través de las culturas no pertenecientes a la europeo-norteamericana para posicionarse desde la exterioridad y fundamentar otro horizonte de vida. 
ahí que sea necesario preguntarse dos cosas: ¿Bajo qué categorías epistemológicas y enfoques se sustenta el desarrollo como sujeto perteneciente al no-ser de la totalidad capitalista?, y ¿qué visión de la educación emanada del nivel de exterioridad del no-ser (locus de enunciación) permitiría la descolonización epistemológica?

La pedagogía de la liberación propone la opción ético-política por la alteridad, es decir, por el/la otro/a excluido/a, explotado/a, dominado/a. Desde esta perspectiva, la enseñanza rompe con las determinaciones que distinguen a la educación modernacapitalista-colonial. En efecto, como el campo pedagógico se encuentra con la realidad de la explotación y la exclusión en América Latina, la alternativa es una enseñanza para la liberación cuyo locus de enunciación defiende la interpelación, la justicia y las luchas de los sujetos oprimidos.

Afirmarse en la alteridad del otro/a hace que la visión de la pedagogía se abra con respecto a la totalidad educativa hegemónica. Así pues, para superar la dominación opresiva se requiere una praxis abierta y dialogante desde y hacia el otro/a. Una visión educativa que permita al excluido enfrentar a la totalidad que genera el escenario de dominación para enfrentarla cara a cara desde su propia materialidad. Esto permite reconocer la concreción del oprimido, identificar su locus de enunciación y defender su pretensión vital de pensar la condición que lo distingue y excluye a partir de un acto comunitario y popular.

En definitiva, posicionarse desde el locus de enunciación latinoamericano significa develar y desnaturalizar la realidad opresora y avanzar a su vez en la liberación del acto educativo para permitir a los/as oprimidos/as pensar, enseñar y exteriorizar otro horizonte de sentido "más allá de la modernidad". La prioridad debe ser, como dirían los pueblos amerindios, la unidad del universo con lo humano y la naturaleza. El encuentro con la idea de sumak kawsay (la vida en plenitud y armonía) en el mundo kuna baluwaba (la unidad de la naturaleza) y en el aymara suma oamaña (el bienestar de tu fuerza interna) es para Bautista la transición hacia esa otra forma de vida, que requiere otra forma de producir conocimiento y sabiduría para la vida.

\section{Referencias}

Bautista, J. J. (2005). Crítica de la razón boliviana. Elementos para una crítica de la subjetividad del boliviano-latino-americano. La Paz: Pisteuma. 
Bautista, J. J. (2014). ¿Qué significa pensar desde América Latina? Madrid: Akal.

Bautista, J. J. (2018), Hacia un Marx del siglo XXI. El problema de la descolonización del pensamiento crítico contemporáneo. En R. Landa (ed.), Vuelo del fénix. El capital: lecturas críticas a 150 años de su publicación (1867-2017) (pp. 121-153). Buenos Aires: Clacso.

Cadahia, L. (2016). Dispositivos estéticos y formas sensibles de la emancipación. Ideas $y$ Valores, 65(161), 267-285.

Díaz, J. A. (2015). Pensamiento crítico en Fals Borda: hacia una filosofía de la educación en perspectiva latinoamericana. Cuadernos de Filosofía Latinoamericana, 36(112), 171-203.

Dussel, E. (1985a). La producción teórica de Marx. Una introducción a los Grundrisse. México: Siglo XXI.

Dussel, E. (1985b). La producción teórica de Marx. Caracas: El Perro y la Rana.

Dussel, E. (1988). Hacia un Marx desconocido. Un comentario de los manuscritos del 61-63. México: Siglo XXI.

Dussel, E. (1990). El último Marx (1863-1882) y la liberación latinoamericana. México: Siglo XXI.

Dussel, E. (1993). Eurocentrismo y Modernidad (Introducción a las Lecturas de Fráncfort). En W. Mignolo (comp.), Capitalismo y geopolítica del conocimiento. Buenos Aires: Ediciones del Signo.

Dussel, E. (1995) Marx y La Modernidad. La Paz: Rincón Ediciones.

Heidegger, M. (2005). ¿Qué significa pensar? Madrid: Trotta.

Hinkelammert, F. (1991). Lucifer y la bestia. Sacrificios humanos y sociedad occidental. San José (Costa Rica): Educa.

Marx, K. (1932). Manuscritos económicos y filosóficos de 1844. México: Siglo XXI.

Mignolo, W. D. (2005). La razón postcolonial: herencias coloniales y teorías postcoloniales. AdVersuS, 2(4), 27-39. 